\title{
A High Resolution Study of the Effect of Morphology On the Mass Spectra of Single PSL Particles with Na-containing Layers and Nodules
}

\author{
Yong Cai, ${ }^{1}$ Alla Zelenyuk, ${ }^{1}$ and Dan Imre ${ }^{2}$ \\ ${ }^{1}$ Pacific Northwest National Laboratory, Richland, Washington, USA \\ ${ }^{2}$ Imre Consulting, Richland, Washington, USA
}

\begin{abstract}
The interpretation and quantification of measurements of particle composition by laser ablation based single particle mass spectrometry is complex. Among the most difficult systems to quantify are internally mixed particles containing alkali metals and organics. The alkali atoms in such particles tend to suppress the formation of other ions sometimes to below the detection limit. Here we present a study of the behavior of single particle mass spectral peak intensities as a function of the amount of the sodium containing compounds deposited on the surface of $240 \mathrm{~nm}$ polystyrene latex (PSL) spheres. We generate three morphologically distinct and well defined coating types: uniform layers, cubic nodules and rounded nodules, and measure the individual particle mass spectra as a function of the vacuum aerodynamic diameter with nanometer resolution. The data show that the probability of detecting the PSL spheres depends on the amount of the alkali metal on the PSL sphere surface, its morphological distribution and the ablation laser power. The data suggest that PSL spheres with localized Na-containing nodules are easier to detect than those which are completely encapsulated. We show, for example, that at low laser power, PSL particles that are completely encapsulated with Na-containing compounds, whose weight fraction is close to $50 \%$, cannot be detected, while $35 \%$ of PSL spheres with same amount of coating can be detected if coating is localized in nodules on a fraction of the particle surface.
\end{abstract}

\section{INTRODUCTION}

Aerosol measurements show that a majority of atmospheric aerosol particles are internally and often heterogeneously mixed

Received 24 January 2006; accepted 8 September 2006.

We thank Dr. Alexander Laskin for his help with the Scanning Electron Microscope. This work was supported by the U. S. Department of Energy Office of Basic Energy Sciences, Chemical Sciences Division. This research was performed in the Environmental Molecular Sciences Laboratory, a national scientific user facility sponsored by the Department of Energy's Office of Biological and Environmental Research at Pacific Northwest National Laboratory (PNNL). PNNL is operated by the US Department of Energy by Battelle Memorial Institute under contract No. DE-AC06-76RL0 1830.

Address correspondence to Alla Zelenyuk, Pacific Northwest National Laboratory, P.O. Box 999, MSIN K8-88, Richland, WA 99354, USA. E-mail: alla.zelenyuk@pnl.gov
(Zhang et al. 2005; Wittig et al. 2004; Lee et al. 2002; Lake et al. 2004; Guazzotti et al. 2001). Aerosol optical properties as well as CCN activity, and thus their behavior and climatic impact, are strongly dependent not only on size and composition, but also on their internal mixing state (Chung \& Seinfeld 2002; Lesins et al. 2002; Jacobson 2000, 2001; Fuller et al. 1999). To this end Single Particle Mass Spectrometry (SPMS) is making very significant contributions at characterizing these properties of atmospheric aerosols. The success of SPMS is, however, presently restricted by its limited quantitative nature. Among the most difficult to quantify, by laser ablation based SPMS, are internally mixed multicomponent particles, especially those containing alkali metal constituents and organics. In the presence of alkali metals the matrix effect in the ablation plume results in charge transfer leading to the suppression of the non-alkali ion constituents. In the worst case, the presence of alkali metal can even result in reduction of the other constituent's signals to below the detection limit. Ion suppression is by no means a unique problem to SPMS in analytical chemistry. In the Matrix Assisted Laser Desorption/Ionization (MALDI) technique, for example, the ion suppression effect is a common phenomenon involving both matrix and analyte (Knochenmuss \& Zenobi 2003).

It is not uncommon that analysis of mixed samples requires either sample separation or application of more than one analytical tool to decipher the composition of the mixture. Since the application of multiple analytical probes to real-time single particle analysis is not a simple matter, it is important to develop an understanding of the processes that determine the relationship between the observed mass spectral intensities and the true particle composition.

It is reasonable to assume that the effect of ion suppression will be dependent on the amounts of the competing elements, their relative electron affinities and the kinetic processes. In order to relate the observed mass spectral ion intensities in multicomponent aerosols to particle composition a few research groups conducted studies designed to measure Relative Sensitivity Factors (RSFs) for specific aerosol components of interest. In a study of internally mixed ultrafine particles composed of $\mathrm{KCl} / \mathrm{NaCl}$ 
and of $\mathrm{NaCl} / \mathrm{NH}_{4} \mathrm{NO}_{3}$, Ge et al. (1998) showed that the respective ion peak area ratios: $\mathrm{Na}^{+} / \mathrm{K}^{+}$and $\mathrm{Na}^{+} / \mathrm{NO}^{+}$can be used to infer particle composition on the basis of empirically derived RSFs for these systems. Similarly a study by Gross et al. (2000) experimentally derived RSFs for alkali metals and ammonium. They showed that their measured RSFs correlate with the ionization potentials and the lattice energies of the alkali metals and can be used to accurately determine the relative amounts of $\mathrm{Na}^{+}$and $\mathrm{K}^{+}$ions in mass spectra of sea-salt particles. However, the extension of this approach to complex multicomponent aerosols has not been tested. Reilly et al. (2000) investigated similar charge transfer induced matrix effects using standard reference materials obtained from the National Institute of Standards and Technology (NIST). They demonstrated that in SPMS ions of major components can be suppressed by species with significantly lower concentrations.

Quantitative analysis of aerosol composition can be additionally complicated by the fact that the mass spectral signal intensity of the particle constituents may depend not only on their relative amounts and RSFs, but also on their morphological arrangement on or in the particle. Despite the importance and abundance of heterogeneously mixed particles the effect of morphology on single particle mass spectra is still poorly understood.

It is well known, for example, that only a fraction of supermicron particles is vaporized and ionized during ablation, resulting in bias toward the composition of the surface of particles. Among a limited number of reports of investigations in this area is a study of the surface composition of micron-sized $\mathrm{KCl} / \mathrm{NaCl}$, $\mathrm{KCl} / \mathrm{KI}$, and $\left(\mathrm{NH}_{4}\right)_{2} \mathrm{SO}_{4} / \mathrm{NH}_{4} \mathrm{NO}_{3}$ particles by Ge et al. (1996). They report that the single particle mass spectra indicate that except at their eutonic points these particles do not have a homogeneous morphology. Carson et al. (1997) report a study of micron-sized $\mathrm{NaCl}$ particles that were exposed to ammonia and nitric acid vapor to produce ammonium nitrate coated $\mathrm{NaCl}$ particles. They found that at low laser irradiance the mass spectra were dominated by the ammonium nitrate coating and at high laser irradiance both $\mathrm{NaCl}$ core and ammonium nitrate coating were ablated.

It is possible to diminish the impact of matrix effects in SPMS by using two lasers to produce ions: an infrared (IR) laser for particle evaporation and a vacuum ultraviolet (VUV) or UV laser to ionize the evaporated plume. Woods et al. (2002) employed such an approach to demonstrate the depth profiling ability of this type of SPMS. They measured mass spectra of micronsized glycerol particles that were coated with oleic acid and showed that at low IR laser powers particles were not completely vaporized resulting in preferential composition analysis of the surface layers, but high IR laser powers evaporated the entire particles producing quantifiable mass spectra representative of the total particle composition.

Commercially available polystyrene latex (PSL) spheres can serve as well defined cores on top of which other substances can be deposited. Excellent examples of studies using such core-shell aerosols were presented by Katrib et al. (2004, 2005). They used PSL spheres coated with oleic acid layers to study the reaction of oleic acid with ozone.

Encapsulated particles are the simplest form of internally mixed morphologically controlled particles that can be generated in the laboratory. Measurement of the mass spectral intensities of the coating and the core as a function of coat thickness provides very valuable information on the relationship between particle morphology and mass spectral signature.

To set the scale for such particle systems, it is worth noting that an application of only a $10 \mathrm{~nm}$ coat to a $200 \mathrm{~nm}$ particle generates a particle whose coating represents $25 \%$ of the particle by volume. To develop an understanding of the behavior of such a mixed system, as a function of the relative composition of the two constituents, clearly requires high spatial resolution. In the present study we use our Single Particle Laser Ablation Time-of-flight mass spectrometer (SPLAT), which can size the particles with the precision of $\pm 0.5 \%$ (Zelenyuk \& Imre, 2005) and clearly satisfies the high resolution requirements.

As reported in Buzorius et al. (2002), SPLAT has already been applied to make measurements of ammonium nitrate (AN) coated PSL particles. In that study SPLAT was coupled with the Humidified Tandem Differential Mobility Analyzer (HTDMA) to measure simultaneously size, composition and hygroscopicity of individual aerosol particles. Buzorius et al. (2002) showed that the hygroscopic properties of PSL spheres coated with AN relate to their coating thickness and mass spectral signatures.

To date each of the aerosol mass spectrometry studies on morphologically controlled particles relied on particles with the homogeneously applied coating over the entire particle. In these systems the interpretation of the experimentally observed peak intensities as function of coating thickness have typically been in terms of penetration depth. No studies of internally mixed particles, in which the different constituents are morphologically separated in a manner that exposes all of them at the particle surface to the laser and the vacuum, have been reported.

We present here a highly resolved study of the effect of morphology on the mass spectra of individual internally mixed particles. In Figure 1 we show a schematic of the three morphologically distinct particle types we produced for this study. They are all composed of $240 \mathrm{~nm}$ PSL spheres coated with Na-containing compounds. Type I is a PSL sphere homogeneously encapsulated with metastable anhydrous glassy $\mathrm{NaNO}_{3}$ (SN). Types II and III are PSL spheres with attached cubic and rounded nodules containing $\mathrm{NaCl}(\mathrm{SC})$ and Sodium Lauryl Sulfate- $\left[\left(\mathrm{CH}_{3}\left(\mathrm{CH}_{2}\right)_{10} \mathrm{CH}_{2}\left(\mathrm{OCH}_{2} \mathrm{CH}_{2}\right)_{2} \mathrm{OSO}_{3} \mathrm{Na}\right]-(\mathrm{SLS})\right.$ respectively.

In each case we measure with nanometer resolution individual particle vacuum aerodynamic diameter, $d_{v a}$, and a mass spectrum using SPLAT. We compare the observations on encapsulated particles to those made on particles with nodules, 


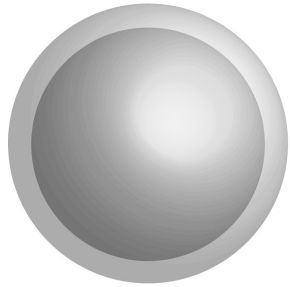

I

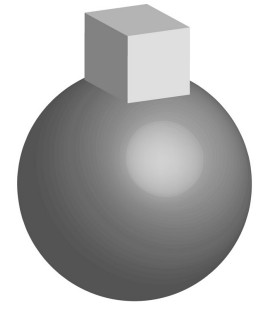

II

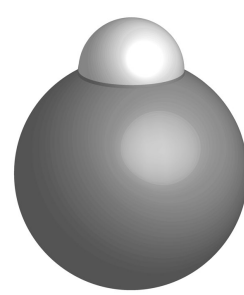

III
FIG. 1. A schematic representation of the three morphologically distinct $240 \mathrm{~nm}$ PSL coated particle types: I are PSL particles coated with metastable glassy $\mathrm{NaNO}_{3}$, II are PSL particles coated with cubic $\mathrm{NaCl}$ nodules, and III are PSL particles coated with the additives, one of which is sodium lauryl sulfate.

in which most of the PSL sphere remains exposed. We show that the presence of sodium can be detected for layers thinner than $1 \mathrm{~nm}$ and that the PSL core signal decays rapidly as a few nanometers of a Na-containing coating are added. Our data indicate that the attenuation of the polystyrene signal does not result from an optically impenetrable sodium containing layer. The data presented here also show that, in all cases the ablation laser evaporates the entire particle, but as a consequence of the processes of charge transfer and ion suppression, at thicker coatings, the carbonaceous ions from the PSL spheres are not detected. We find a difference between the behavior of PSL particles and particles with localized sodium, which we attribute to morphology.

\section{EXPERIMENTAL}

A water solution containing $240 \mathrm{~nm}$ PSL spheres (Duke Scientific) and either SN or SC is aerosolized using an atomizer (TSI Inc., Model 3076), and dried with two inline diffusion dryers (TSI Inc., Model 3062). The nearly dry aerosol flow proceeds to a mixing and dilution chamber, where the relative humidity is reduced to below $1 \%$. This particle generation and drying method leads to the production of two types of particles: salt particles and PSL particles "coated" with salt. The dry aerosol is classified with a DMA (TSI Inc., Model 3081) to produce a sample with a narrow mobility distribution prior to sampling by SPLAT. Pure salt particles are distinguished from coated PSL on the basis of their aerodynamic diameters, taking advantage of the differences in densities. The drying conditions and concentrations are chosen on the basis of our previous studies (Zelenyuk et al. 2005; Zelenyuk et al. 2006).

Previous studies of pure SN particles (Zelenyuk et al. 2005), in which the density of particles at near zero RH was measured, showed that SN forms persistent droplets. These observations are consistent with Hoffman et al. (2004), where micron-sized SN particles were observed microscopically to form dropletlike structures even under vacuum and when placed in contact with Transmission Electron Microscope (TEM) grids. Tang and
Munkelwitz (1994) have studied the behavior of individual, suspended, $\sim 10$ micron SN particles as a function of RH. They also concluded that SN particles do not effloresce. The microscopic images we present in the section below provide support for the fact that the SN coat remains in glassy/liquid form even when in contact with the PSL sphere.

Unlike SN, sodium chloride particles effloresce at $\sim 40 \% \mathrm{RH}$ forming particles that are almost cubic in shape. In the presence of the PSL spheres SC forms localized cubic nodules, one or very rarely two per particle.

The third coating type that produces PSL particles with Nacontaining nodules is generated by aerosolizing a more concentrated water suspension of $240 \mathrm{~nm}$ PSL particles containing additives, one of which is SLS. During drying, the SLS deposits together with other additives on the surface of the PSL spheres forming localized round nodules, one or very rarely two per particle.

A detailed description of SPLAT is given in Zelenyuk and Imre (2005); here we give a brief description only. Particles enter the instrument through a 100 micron orifice into an aerodynamic lens inlet. The lens forms a very narrow low divergence particle beam and transmits the particles into the vacuum chamber with high efficiency. As the particles pass through the lens they acquire velocities that depend on of their aerodynamic diameters. From the lens, particles pass through two differentially pumped stages and enter the main chamber, which is equipped with two optical detection stages positioned $16 \mathrm{~cm}$ apart. In each optical detection stage a particle crosses a green laser beam and the scattered light it generates is collected by an ellipsoidal reflector and detected by a photomultiplier. Each particle is detected twice, once at each stage, and the time of flight between the two optical detection stages is recorded. Measuring the time of flight of PSL particles of known diameter and density calibrates the time of flight and aerodynamic diameter measurements. Once detected and sized, particle composition is obtained by UV laser ablation and time-of-flight mass spectrometry. To probe for the relationship between laser power and particle mass spectral signatures measurements were conducted at two laser powers for each particle type. The high and low laser powers correspond to the laser fluences of $5 \pm 0.5 \mathrm{~J} / \mathrm{cm}^{2}$ and $2.5 \pm .25 \mathrm{~J} / \mathrm{cm}^{2}$, respectively for an excimer laser operated at $193 \mathrm{~nm}$ with $15 \mathrm{~ns}$ pulse length.

In some experimental runs the aerosol flow from the DMA is simultaneously sampled by SPLAT and collected onto copper TEM grids to obtain high vacuum images using Scanning Electron Microscope (SEM). Detailed description of the SEM setup and the Time Resolved Aerosol Collector (TRAC) was presented by Laskin et al. (2005).

The mass spectral data are analyzed by integrating the mass spectral intensities of the peaks assigned to polystyrene, $I_{P S L}$, and coating substance, $I_{\text {coat }}$, respectively and by examining their behavior as a function of coating thickness and laser power. Coating thickness is calculated using the relationship between the particle vacuum aerodynamic diameter, $d_{v a}$, and the coating 
thickness, $\delta$, given by Equation (1):

$$
d_{v a}=\frac{d_{0}^{3} \rho_{P S L}+\left[\left(d_{0}+2 \delta\right)^{3}-d_{0}^{3}\right] \rho_{c}}{\left(d_{0}+2 \delta\right)^{2} \rho_{0}}
$$

Where: $d_{0}=240 \mathrm{~nm}$ is $\mathrm{PSL}$ spheres' diameter, $\rho P S L=$ $1.05 \mathrm{gcm}^{-3}$ is the density of ${ }_{\mathrm{PSL}}$ spheres, $\rho_{c}$ is density of the coating material $\left(2.10 \mathrm{gcm}^{-3}\right.$ for $\mathrm{NaNO}_{3}$ and $2.165 \mathrm{gcm}^{-3}$ for $\mathrm{NaCl}$ ), and $\rho_{0}=1 \mathrm{gcm}^{-3}$ is the unit density. Equation (1) assumes the particles to be spherical with a homogeneous and uniformly distributed coating. For particles with SC nodules coating thickness is a calculated quantity that can be thought of as an approximately equivalent coat thickness. In other words it is the thickness of a coating that is evenly distributed over the particle that would have the same vacuum aerodynamic diameter. This interpretation ignores the small effects we may have due to a dynamic shape factors which, for the particles involved we estimate, on the basis of our previous studies (Zelenyuk et al. 2006) to be below 1.15.

\section{RESULTS AND DISCUSSION}

\section{PSL Particles Encapsulated with Metastable Glassy Sodium Nitrate}

$\mathrm{SN}$ is one of a few hygroscopic salts that tend to form metastable persistent droplets even at close to zero RH. Once anhydrous SN droplets are formed they do not crystallize even when in contact with a solid surface such as the TEM grids.

The microscopic image presented in Figure 2 is of particles collected on TEM grid after they have been dried and classified by the DMA at $280 \mathrm{~nm}$ mobility diameter. Two types of particles are apparent: two pure SN particles and a PSL particle that is coated with $\mathrm{SN}$. We find that pure SN particles wet the substrate forming what looks like flat droplets of larger diameter. The 240 nm PSL sphere surrounded by a SN coat shows no indication of crystallization. We have examined a large number of images of both particle types and found none of the observed particles to be crystalline.

Figure 3 shows three of the measured $d_{v a}$ distributions for runs in which the DMA was set to select particles with 240 $\mathrm{nm}, 260 \mathrm{~nm}$, and $280 \mathrm{~nm}$. For each DMA setting two peaks are observed: the one with smaller aerodynamic diameter (shaded) corresponds to coated PSL particles and the second to pure SN.

The pure SN peaks positions yield a density of $2.12 \mathrm{gcm}^{-3}$, which is in excellent agreement with our previous measurements (Zelenyuk et al. 2005). An examination of the $d_{v a}$ distributions in Figure 3 shows that the size distributions of the coated PSL particles are significantly broader than those of pure sodium nitrate despite the fact that the two are simultaneously selected by the DMA. The broader size distribution is a reflection of the fact that the density of the coated particles is a function of their size. When transforming the original mobility distribution, whose width is determined by the DMA operating conditions, to the aerodynamic size distribution it is important to take into

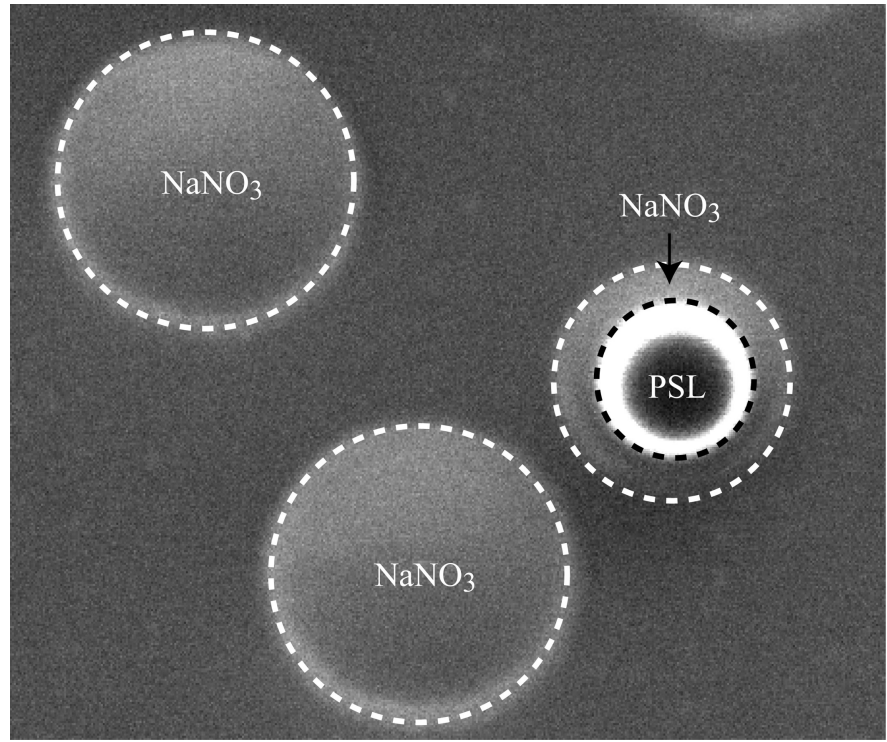

FIG. 2. Annotated Scanning Electron Microscope (SEM) images of a PSL particle coated with $\mathrm{NaNO}_{3}$ and two pure $\mathrm{NaNO}_{3}$ particles, all with $280 \mathrm{~nm}$ mobility diameters. These images show that $\mathrm{NaNO}_{3}$ does not crystallize even under vacuum in the SEM despite the presence of either the TEM grid or the PSL particle. The image shows that $\mathrm{NaNO}_{3}$ remains in a liquid-like phase that wets the TEM grid surface.

account the fact that particles with smaller mobility diameters have a thinner coating and hence lower density, while particles with larger mobility diameters have thicker coating and also higher density. For example, SN coated PSL particles with

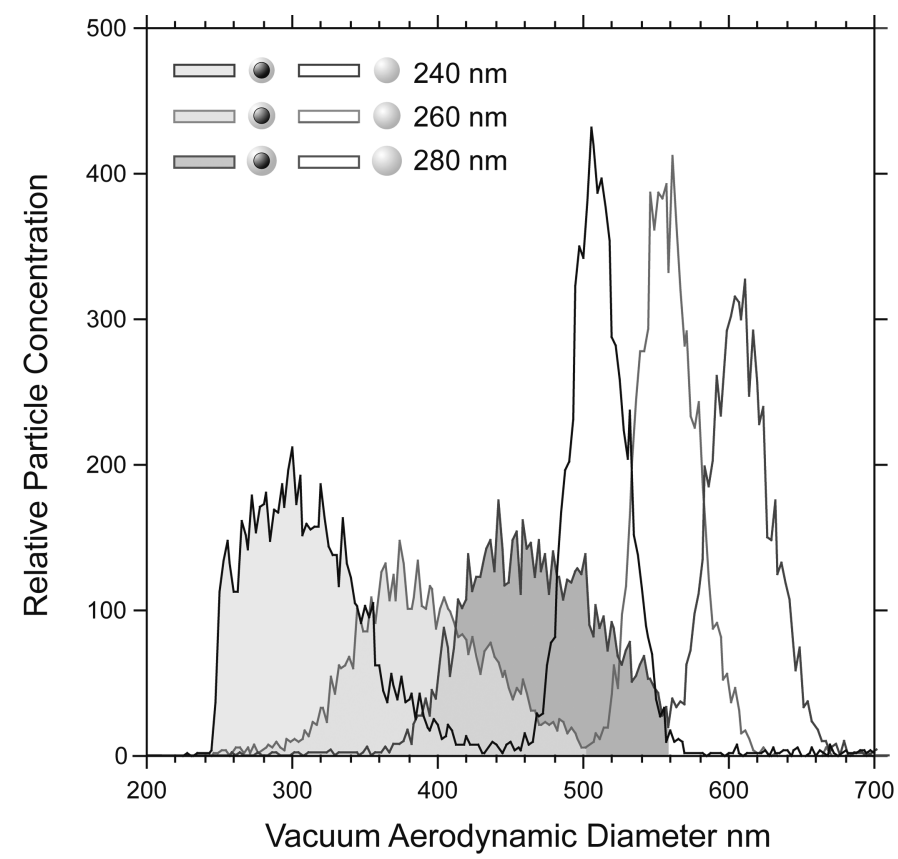

FIG. 3. Three of the observed vacuum aerodynamic size distributions of aerosols selected by the DMA at $240 \mathrm{~nm}, 260 \mathrm{~nm}$, and $280 \mathrm{~nm}$ and composed of pure $\mathrm{NaNO}_{3}$ particles (open) and PSL particles coated with $\mathrm{NaNO}_{3}$ (shaded). 
mobility diameter of $260 \mathrm{~nm}$ and a distribution width of $8 \%$ FWHM (full width at half maximum) should yield a $d_{v a}$ distribution with a width of $24 \%$ (FWHM), which is in good agreement with our observations. Finally we note in Figure 3 that the sharp cutoff on the smaller particle diameter side coincides perfectly with the pure $240 \mathrm{~nm}$ PSL particles $d_{v a}$ distribution.

Figure 4 shows three separate mass spectra of SN coated PSL particles and one of pure SN particles. Spectra $4 \mathrm{a}, 4 \mathrm{c}$, and $4 \mathrm{~d}$ were taken at high laser power and $4 \mathrm{~b}$ represents a run at low laser power. Note that despite the fact that the mass spectra in Figures $4 \mathrm{a}$ and $4 \mathrm{~b}$ are of particles with an average SN coat thick-

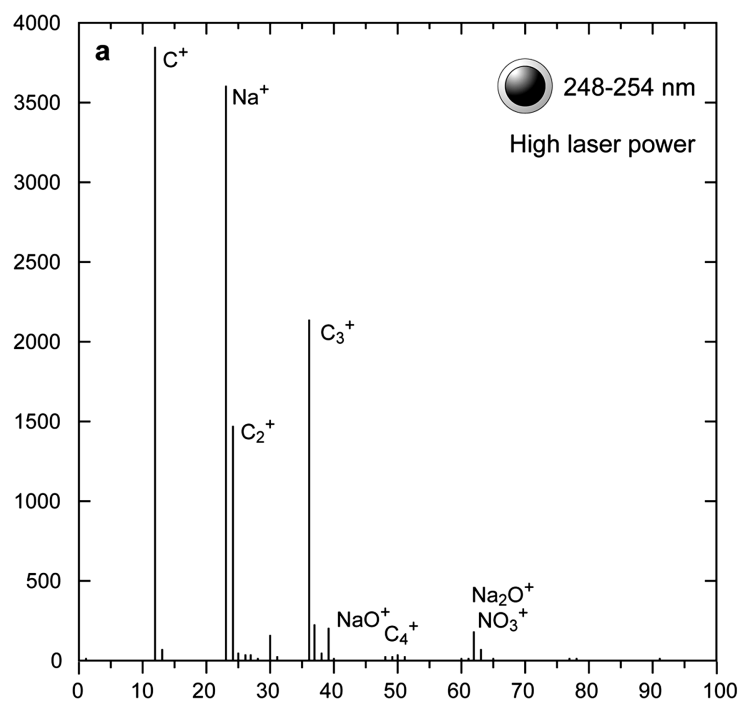

AMU

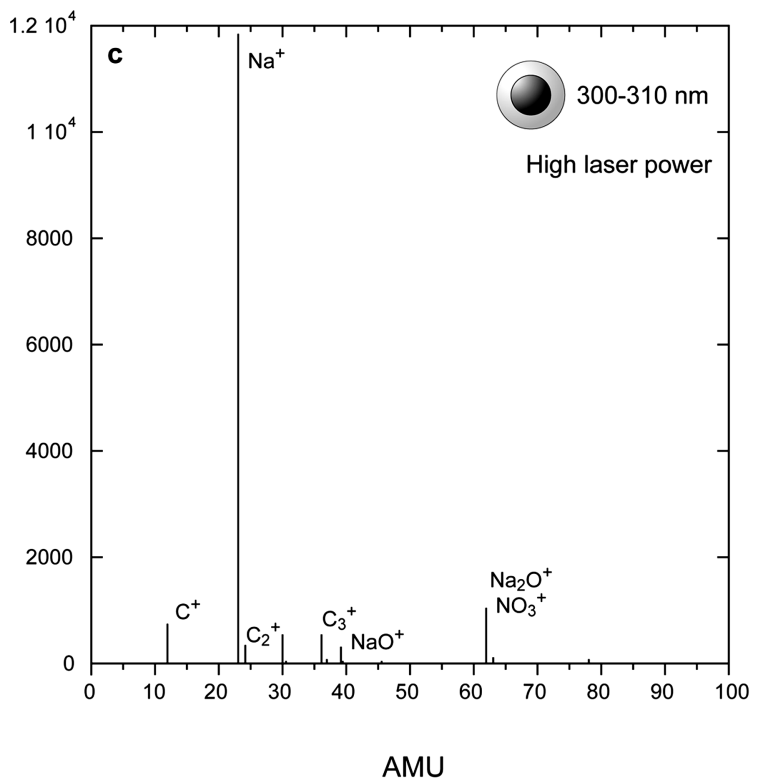

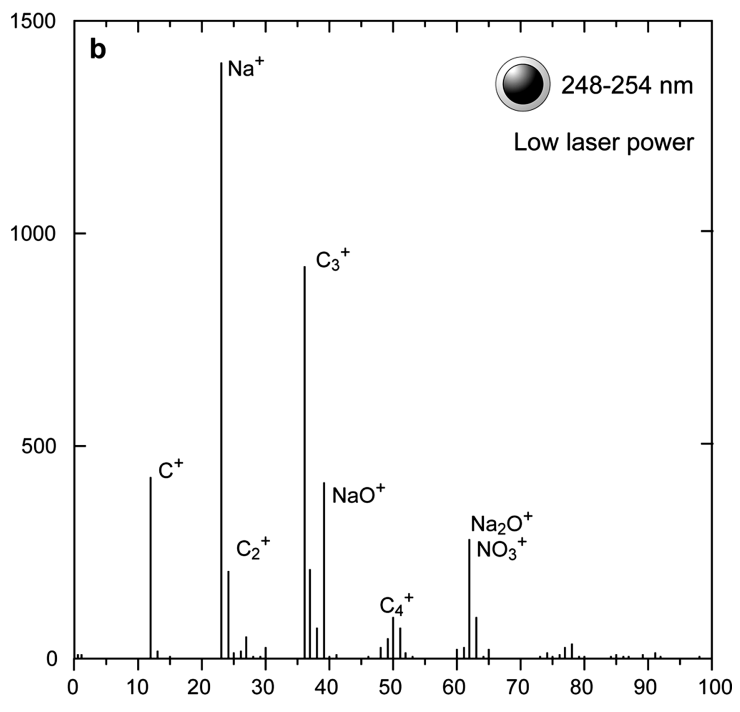

ness that is nearly zero the SN peak intensities are significant. A comparison between the spectra in Figures $4 a$ and $4 b$ reveals that at lower laser powers the relative contribution from the SN coating is significantly higher. This is a reproducible trend observed for all the systems and all coating thicknesses presented in this paper. A second notable and reproducible feature is that at lower laser power mass spectra exhibit a significant decrease in fragmentation.

The mass spectrum in Figure 4c, which is clearly dominated by $\mathrm{SN}$, was generated by averaging spectra of particles with a $\mathrm{SN}$ coat that is 6 to $7 \mathrm{~nm}$ thick. The last mass spectrum is of pure

AMU

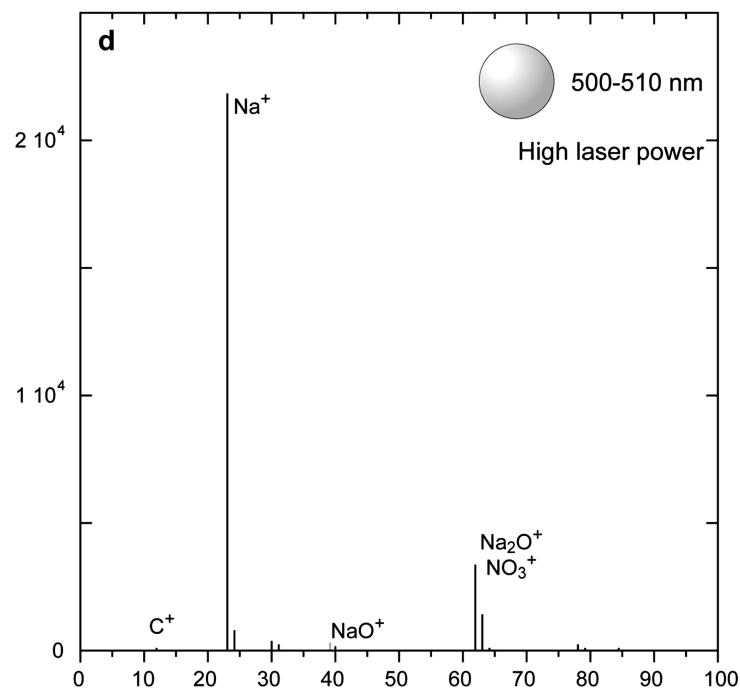

AMU

FIG. 4. Mass spectra of PSL particles coated with $\mathrm{NaNO}_{3}$ and pure $\mathrm{NaNO}_{3}$ particles generated from the same solution. Spectra (a) and (b) were obtained by averaging individual particle mass spectra of coated PSL particles with vacuum aerodynamic diameters that cover the size distribution of pure PSL particles and were taken at high and low laser powers respectively. Spectrum (c) corresponds to larger particles with thicker coating and (d) is an average mass spectrum of pure sodium nitrate particles from the very same solution. 


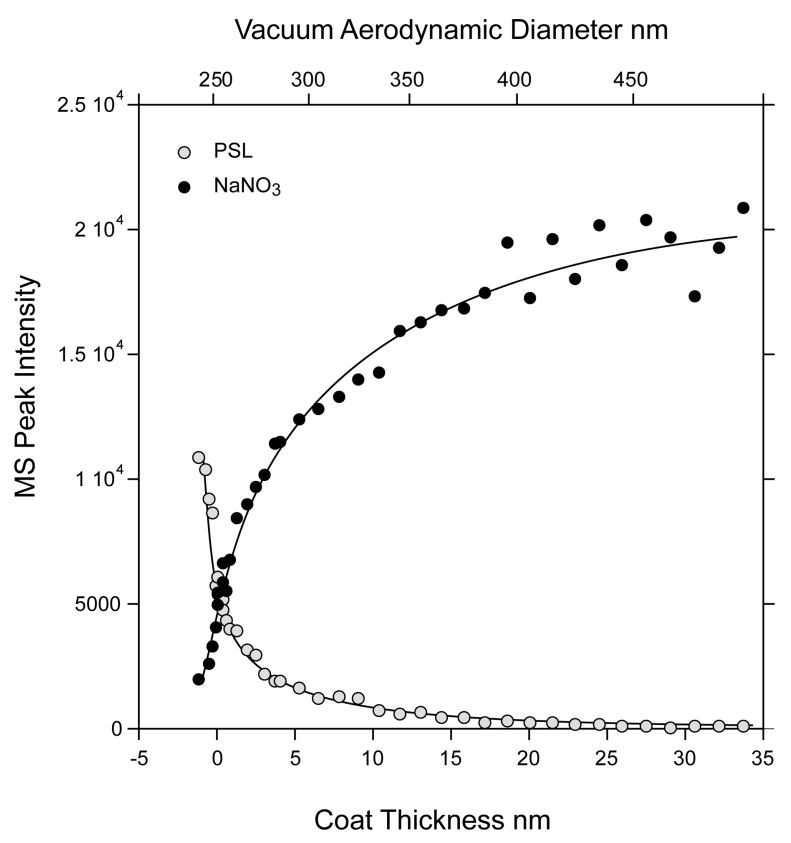

FIG. 5. Polystyrene and $\mathrm{NaNO}_{3}$ Mass Spectral (MS) peak intensities of coated PSL particles as a function of either $d_{v a}$ (top scale) or of the thickness of the $\mathrm{NaNO}_{3}$ coat (bottom scale).

SN particles generated from the very same solution. It exhibits a very small amount of $\mathrm{C}^{+}$ions whose origin is the manufacturer additives in the PSL particle suspension. We use the intensity of this peak to establish a background level.

Figure 5 shows a plot of the sums of the PSL core and sodium nitrate mass spectral peak intensities measured at high laser power and plotted as a function of the SN coat thickness and the measured $d_{v a}$. The most striking aspect of these data is the rapid rate with which the PSL signature diminishes and the concomitantly rapid increase of the SN intensity, such that, a $30 \mathrm{~nm}$ thick layer of SN reduces the PSL signature nearly to background level at high laser powers, while $15 \mathrm{~nm}$ of SN are sufficient to achieve the same at low laser power. Note that while layers of $15 \mathrm{~nm}$ and $30 \mathrm{~nm}$ seem to be thin, they correspond to particles composed of $46 \%$ and $66 \%$ of SN by weight respectively.

One can define a PSL detection probability, based on the fraction of particles with the PSL mass spectral signature (IPSL) above a threshold. Note that this detection efficiency depends on a somewhat arbitrarily chosen minimum threshold that signifies the presence of a PSL particle. We set this minimum such that fewer than $10 \%$ of "pure" SN particles, which are generated from the very same solution and thus have minor carbon ion peak as shown in Figure 4d, would wrongly be classified as coated PSL particles. We find that at low laser power, SN coated PSL particles are indistinguishable from pure SN particles when the coat thickness reaches $15 \mathrm{~nm}$. At twice the laser power $40 \%$ of PSL containing particles can still be detected despite of the presence of a $30 \mathrm{~nm}$ thick $\mathrm{SN}$ coat.

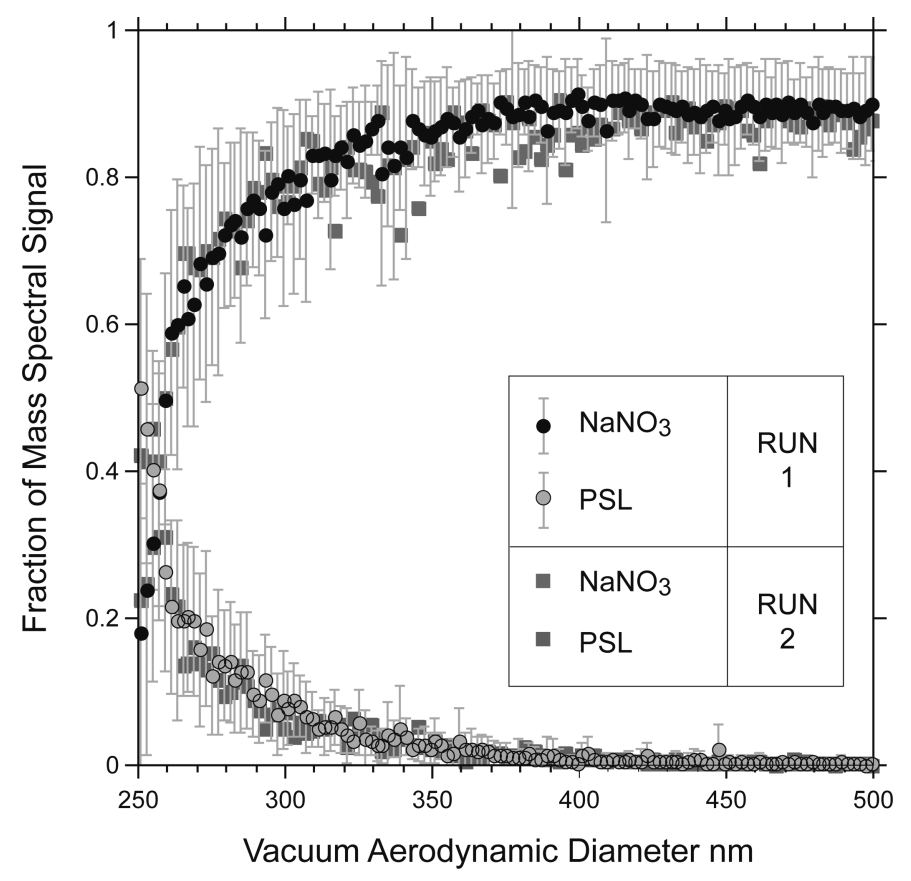

FIG. 6. A statistical analysis of the noise and reproducibility of the mass spectral peak intensities in a plot of relative mass spectral signal intensities for PSL and $\mathrm{NaNO}_{3}$ for two separate experimental runs 1 and 2 . The laser power for the two runs differed by $\sim 10 \%$. The error bars are associated with run 1 only and indicate $2 \sigma$.

Noise and Reproducibility of Observed Data for PSL Particles Encapsulated with Metastable Glassy Sodium Nitrate

Before we proceed to the next two systems it is important to set the scales for noise and reproducibility. Here noise refers to the repeatability of individual particle mass spectral peak intensities during $a$ run and reproducibility relates to a comparison between calculated average mass spectral peak intensities for two runs that were carried out a number of days apart with new solutions and laser realignment.

The absolute mass spectral peak intensities vary from one particle to the next significantly more than an individual particles' relative mass spectral peak intensity. We therefore resort to analyzing the relative peak intensities since it clearly removes one of the important noise sources. A summary of the statistical analysis is presented in Figure 6. The number of particles contributing to the average value given in each of the presented 2 $\mathrm{nm}$ slices varies between 8 and $\sim 100$. Figure 6 presents datasets for two different runs. Run 1 includes averaged fractional mass spectral values and the standard deviations $(2 \sigma)$. We conclude that 8 particles per bin are sufficient to yield statistically significant values. A comparison between the data obtained by the two runs, which were carried out under similar conditions, with the ablation laser operated at only $10 \%$ difference in laser power, clearly demonstrates that it is possible to generate quantitatively meaningful data with very high spatial resolution. Analysis of the data presented in the following sections produced very similar results and was therefore not included. 


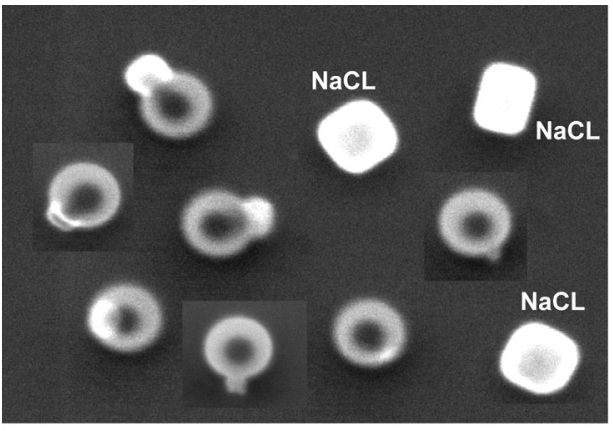

$240 \mathrm{~nm}$

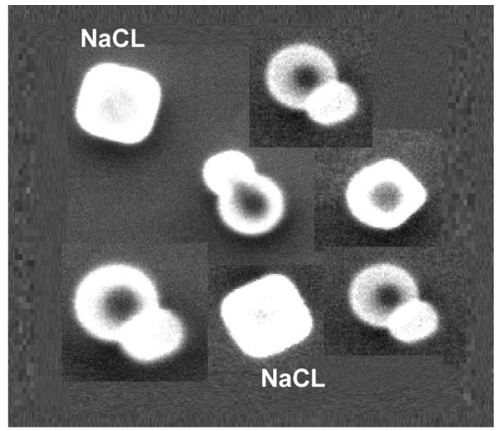

$280 \mathrm{~nm}$

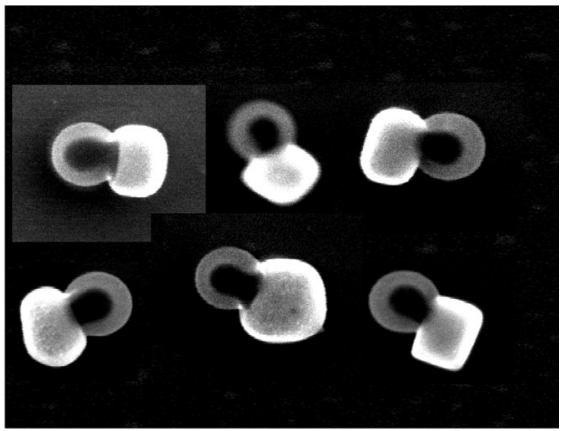

$320 \mathrm{~nm}$

FIG. 7. SEM images of PSL particles coated with $\mathrm{NaCl}$ and $\mathrm{NaCl}$ particles (labeled) of three mobility diameters as indicated. These images provide evidence that the $\mathrm{NaCl}$ forms nearly cubic nodules of a wide range of sizes on the PSL particles.

\section{PSL Particles with Cubic NaCl Nodules}

In a recently completed study (Zelenyuk et al. 2006) on the dynamic shape factor of sodium chloride particles we found that atomization of an aqueous SC solution can produce three different forms of crystalline SC particles: cubic, nearly spherical, and agglomerated. On the basis of that experience we chose for the present study the drying conditions that produce cubic SC particles.

Figure 7 shows three sets of micrographs of DMA classified SC and SC coated $240 \mathrm{~nm}$ PSL spheres. The micrographs show that $\mathrm{NaCl}$ does indeed form nearly cubic nodules that are attached to PSL spheres. As a result of the $\sim 8 \%$ FWHM distribution in mobility diameters there is a noticeably wide distribution in the size of the $\mathrm{NaCl}$ nodules, but the overall trend of increasing nodule size with mobility diameter is clearly visible.

Figure 8 shows the observed $d_{v a}$ distributions for three separate measurements with the DMA set to select particles with 240 $\mathrm{nm}, 260 \mathrm{~nm}$ and $280 \mathrm{~nm}$ mobility diameters. For each DMA setting two peaks were observed: the one with smaller $d_{v a}$ (shaded) corresponds to PSL particles with SC cubic nodules and the second to pure SC. The pure SC peak positions yield an effective density of $1.87 \mathrm{gcm}^{-3}$, in good agreement with our previous measurements (Zelenyuk et al. 2006). Comparing the observed $d_{v a}$ distributions for $\mathrm{SC}$ and $\mathrm{SN}$ reveals a qualitative difference for the DMA setting of $240 \mathrm{~nm}$ but not for others. The $\mathrm{SN} d_{v a}$ distributions of coated PSL particles indicate that the size selected aerosol contains comparable number concentrations of particles with $d_{v a}$ in the range from $\sim 250$ to $\sim 340 \mathrm{~nm}$. In contrast the $d_{v a}$ distributions of SC coated PSL particles seemingly show a rapid decrease in the concentrations of coated particles with vacuum aerodynamic diameters larger than $\sim 260 \mathrm{~nm}$. Since the concentration of SC was only 1.5 times lower than the corresponding SN concentration it cannot account for the observed large differences. It is more likely that the rapid drop in the number of detected $\mathrm{NaCl}$ coated PSL particles with larger $d_{v a}$ is a result of a decrease in the aerodynamic lens transmission efficiency for these aspherical particles. It is consistent with our previously reported results, where we observed decreases on the order of a factor of 10 in detection probability as a result of particle deviation from sphericity (Zelenyuk \& Imre 2005).

Figure 9 show two mass spectra of SC coated PSL particles acquired at high laser power. Spectrum $9 \mathrm{~b}$, which is dominated by an intense sodium ion peak, corresponds to particles with an average equivalent SC coat thickness of 6 to $7 \mathrm{~nm}$. As in the case of $\mathrm{SN}$, the $\mathrm{Na}^{+}$peak is relatively more intense in the spectra that were acquired at low laser power (not shown)

Figure 10 shows plots of the fraction of mass spectral signal intensity that has been assigned to the PSL core as a function of three related parameters: particle vacuum aerodynamic diameter

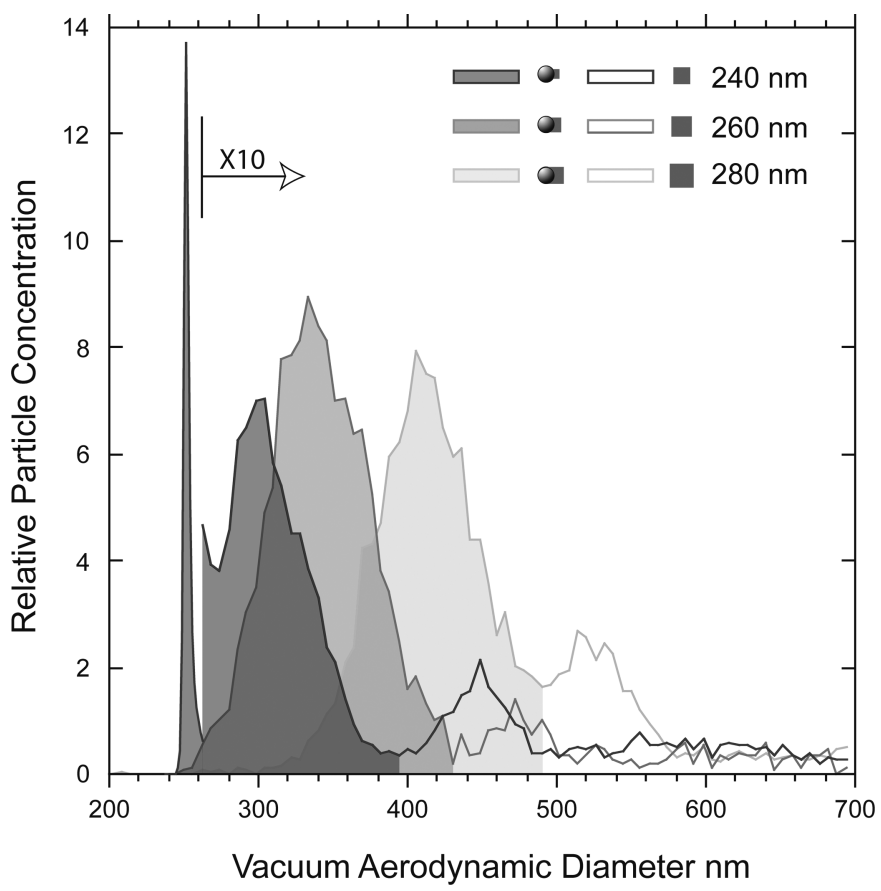

FIG. 8. Three of the measured $d_{v a}$ distributions of aerosols composed of $\mathrm{NaCl}$ particles (open) and PSL particles coated with $\mathrm{NaCl}$ nodules (shaded) and selected by the DMA at $240 \mathrm{~nm}, 260 \mathrm{~nm}$, and $280 \mathrm{~nm}$. Note a factor of 10 change in sensitivity. 

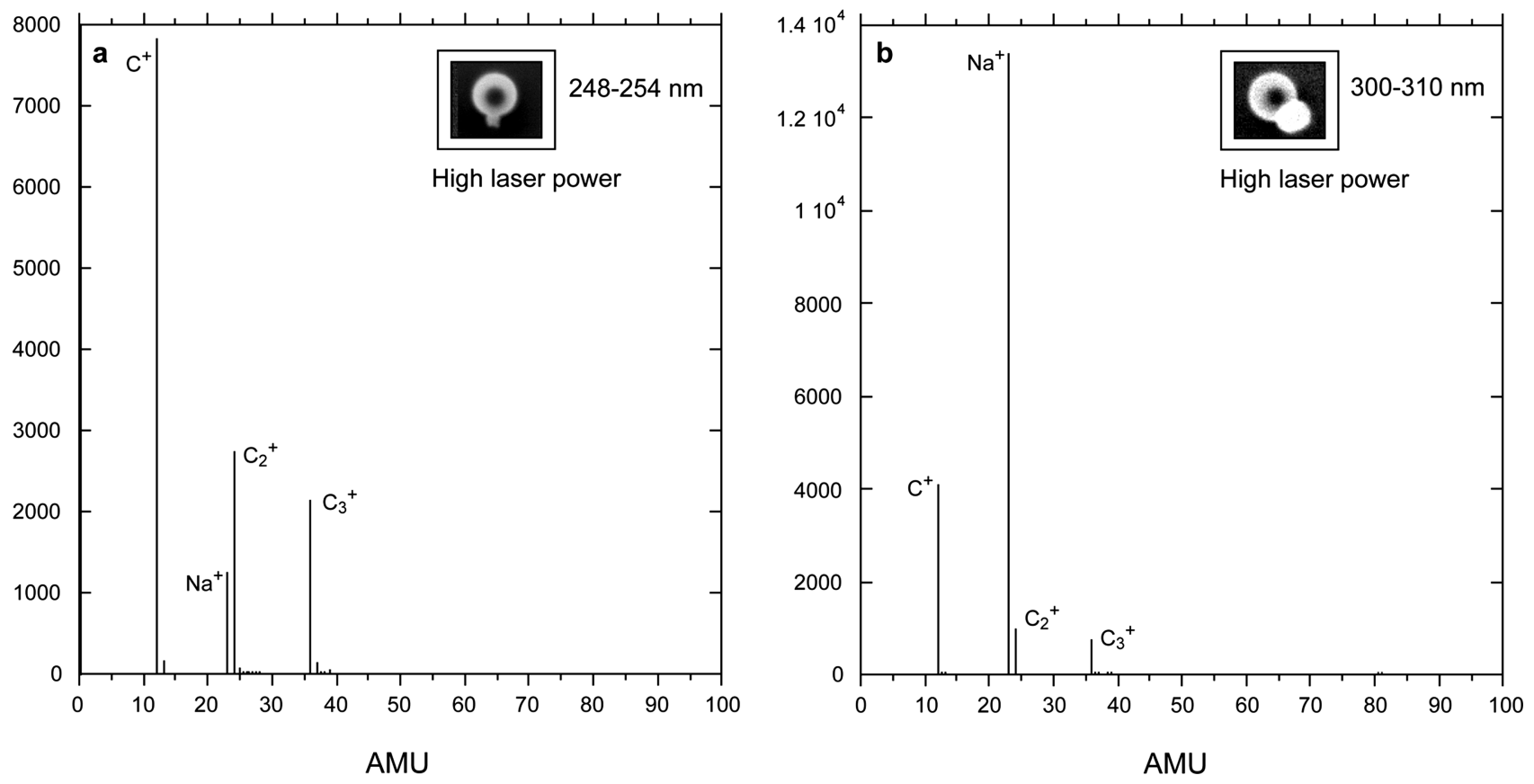

FIG. 9. Mass spectra of PSL particles with $\mathrm{NaCl}$ nodules obtained by averaging the mass spectra of coated particles with vacuum aerodynamic diameters that: (a) cover the size distribution of pure PSL particles and (b) are 300-310 nm, all taken at high laser powers.
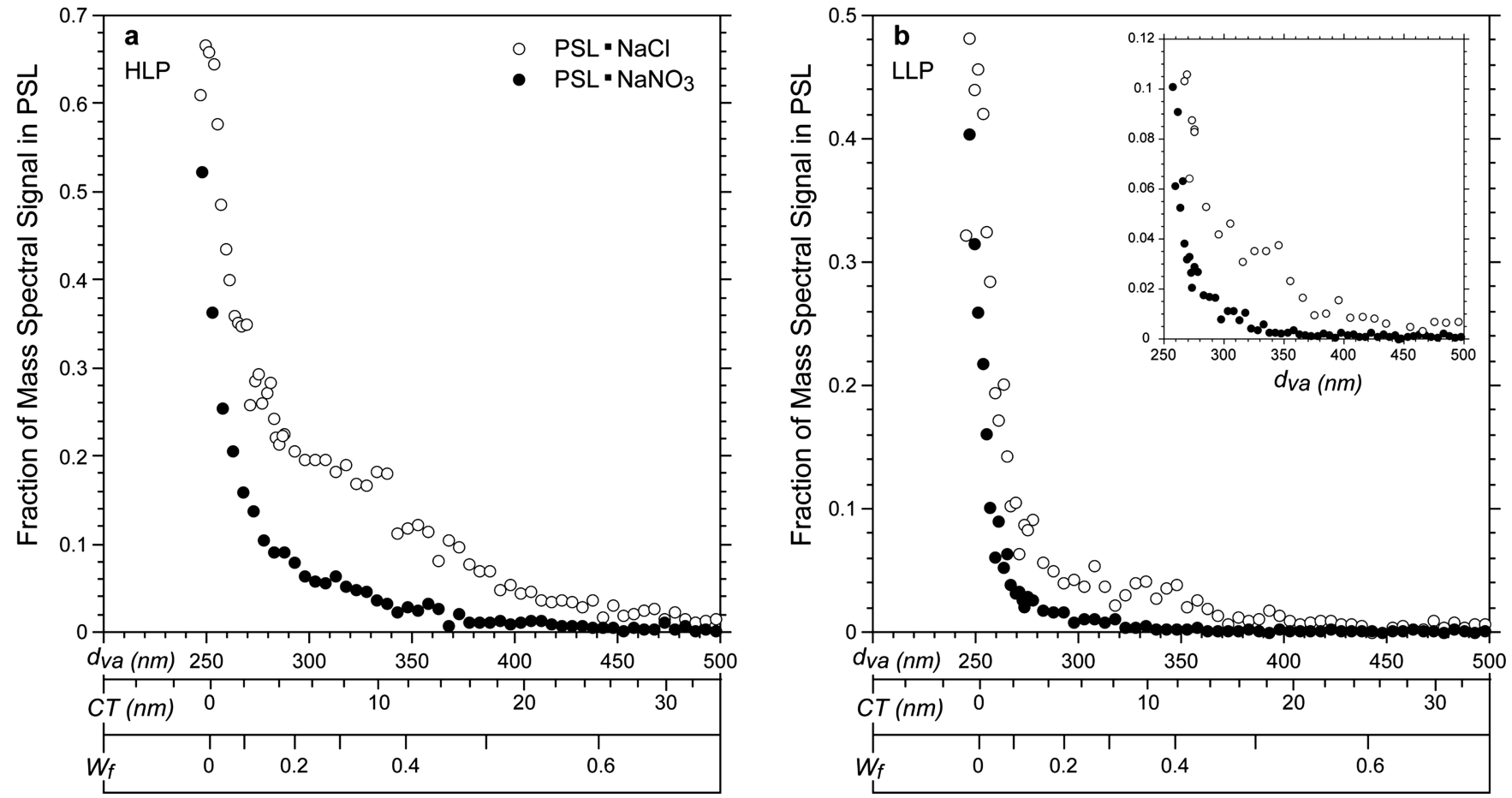

FIG. 10. Plots of the fraction of observed PSL mass spectral peak intensities for $\mathrm{NaCl}$ and $\mathrm{NaNO}_{3}$ coated PSL particles, at two laser powers (high and low laser powers marked HLP and LLP in the figure), as a function of three related variables: $d_{v a}$-vacuum aerodynamic size, $C T$ - equivalent coat thickness, and Wf-coating weight fraction. 
( $d_{v a}$ in $\mathrm{nm}$ ), equivalent coat thickness (CT in $\mathrm{nm}$ ) and the weight fraction of the coating (Wf) for SN and SC. While the $d_{v a}$ is directly measured the other two variables are estimated with the approximations of uniformly distributed coating with density of $2.13 \mathrm{gcm}^{-3}$, which is the average density of sodium nitrate $\left(2.10 \mathrm{gcm}^{-3}\right)$ and sodium chloride $\left(2.165 \mathrm{gcm}^{-3}\right)$. These simplifying approximations are justified as long as these scales are used to provide approximate context for the observed behavior of the mass spectral peak intensities.

As seen from the figure the PSL mass spectral signal is rapidly decays with the addition of SC. A comparison between the data for SC to SN coated PSL particles shows that the PSL signal for the former decays slower with differences between the two coating types being more pronounced at high laser powers. The observed differences between SN and SC coated PSL particles suggest that the coating morphology could be playing a role. PSL particles with SC nodules have a significant fraction of the PSL sphere exposed, making escape of PSL fragment ions in the ablation plume more likely than in case of the particles that are entirely encapsulated by SN.

Figure 11 shows a comparison between the detection probabilities of the PSL spheres for SN and SC coated PSL particles as a function of the same three parameters we used in Figure 10.

\section{Vacuum Aerodynamic Diameter nm}

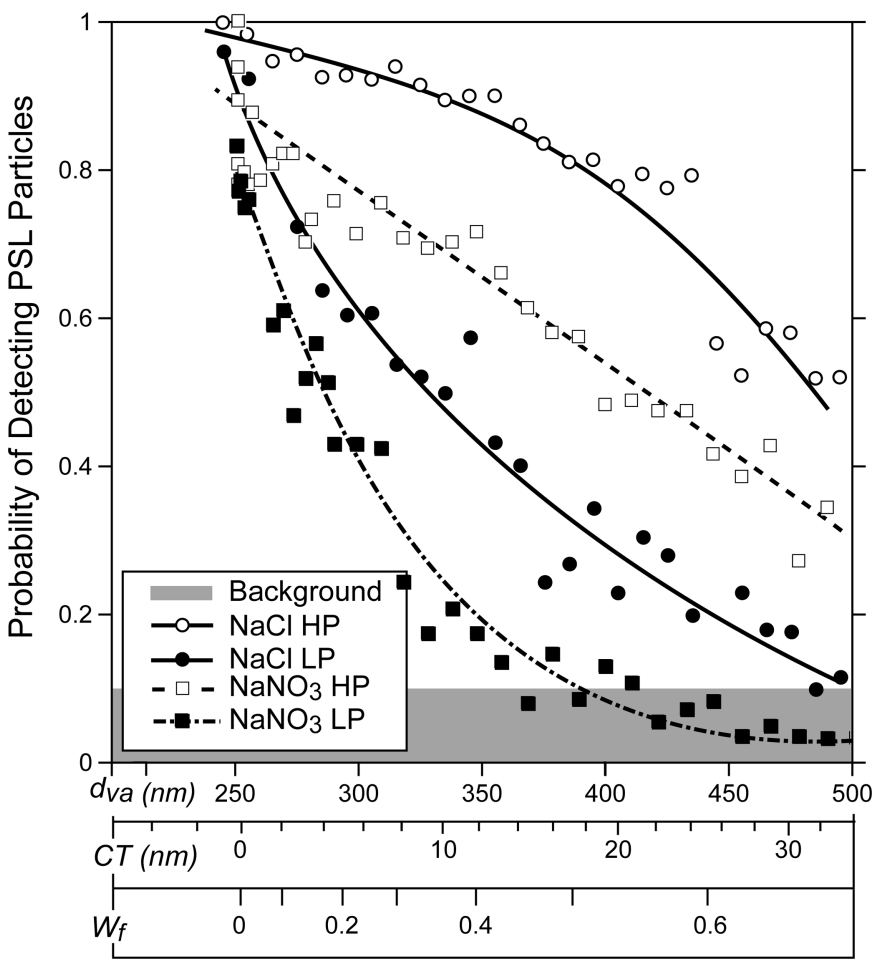

FIG. 11. A plot of the PSL particle detection probability for $\mathrm{NaCl}$ and $\mathrm{NaNO}_{3}$ coated PSL particles at two laser powers as a function of three related variables: $d_{v a}$-vacuum aerodynamic size, $C T$ - equivalent coat thickness, and $W f$ coating weight fraction.
We find that the probability of detecting PSL particles with SC nodules, in which a significant fraction of the PSL particle is exposed, is higher than that found for particles encapsulated with $\mathrm{SN}$.

\section{What Fraction of Each Particle Is Being Evaporated?}

A number of experimental and theoretical studies attempted to determine how much of the individual particle is evaporated in the process of ablation (Carson et al. 1997; Weiss et al. 1997; Schoolcraft et al. 2000; Vera et al. 2005). Thus far the data indicate that the ablation of micron-sized particles results in incomplete particle evaporation. In contrast, Ge et al. (1998) showed that ablation of $\mathrm{KCl} / \mathrm{NaCl}$ particles with diameters around 60 $\mathrm{nm}$ results in complete particle evaporation. A very recent study by Vera et al. (2005) used measurements of initial velocity distributions of ions generated by laser ablation of individual PSL particles in the size range from 196 to $1495 \mathrm{~nm}$ to deduce that in their system the evaporation of particles of all sizes was incomplete. The study by Vera et al. (2005) used an ablation laser with a wavelength of $337 \mathrm{~nm}$ that was operated at $300 \mu \mathrm{J} /$ pulse, a pulse duration of $3 \mathrm{~ns}$ and a focal spot diameter of $\sim 30 \mu \mathrm{m}$. It is important to keep in mind that since ablation is a multiphotondriven process that strongly depends on the electronic absorption spectra of the ablated material it is expected to be highly dependent not only on laser power density but also on the laser wavelength. We will show below that the observations presented in this manuscript suggest that under our experimental conditions all the particles in this study are completely evaporated.

The data presented in the SN section above exhibit a rapid decrease of the PSL core signal as a function of the SN coating thickness. Alone these data can be interpreted to suggest that the ablation of particles with thicker coatings evaporates only a thin layer near the particles' surface and that thicker coatings prevent access to the PSL core. However, the observed behavior of the PSL particles with SC nodules does not support such interpretation. An examination of the microscopic images of these particles in Figure 7 clearly reveals that the surface of the PSL cores are mostly exposed even for the particles with the largest SC nodules. Yet, in these particles the PSL core mass spectral peak intensities decays rapidly with increasing SC nodule size. We would expect the mass spectra to be more reflective of the particles' surface when the laser power is insufficient to evaporate the entire particle. The fact that the data show that the differences in the behavior of the PSL mass spectral peak intensities, between SC and SN coated PSL particles are smaller at lower laser power indicates that for both particle systems, even at low laser power, the ablation laser must consistently evaporate the entire particle.

Moreover, if we were to assume that the only species present in the mass spectrum originate from the ablated particle surface we would expect a fraction of SC coated PSL particles to exhibit significant mass spectral signal intensity from the PSL core for all coat thicknesses. And this effect would be expected to be more pronounced for low laser powers. Since our observations 

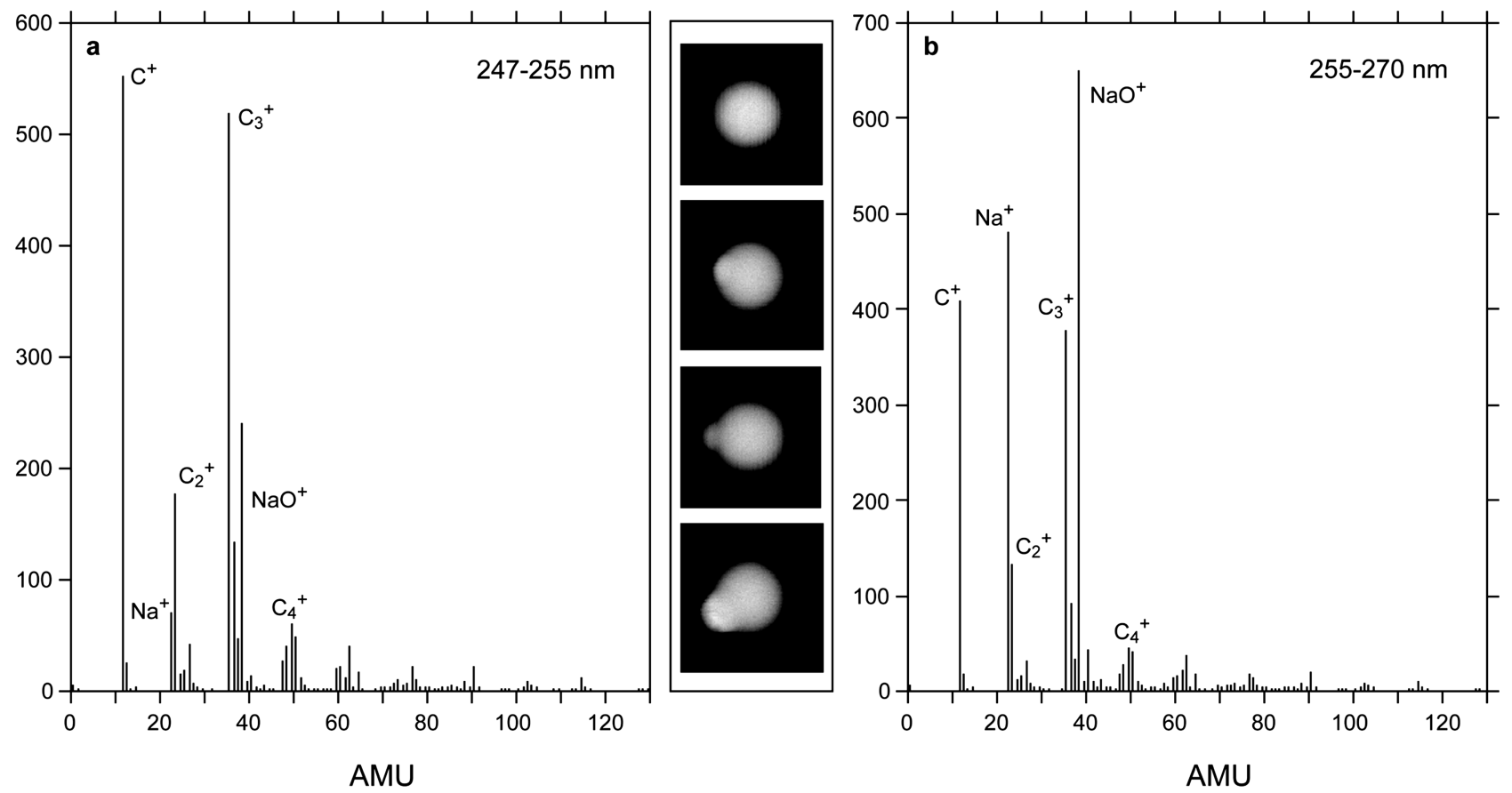

FIG. 12. Two mass spectra of PSL particles coated with SLS nodules. Spectra (a) and (b) were obtained by averaging mass spectra of PSL particles with vacuum aerodynamic diameters that cover the size distribution of pure PSL particles and particles with larger diameters respectively. Both spectra were recorded at low laser power. The SEM images between the two spectra clearly show the presence of rounded nodules.

clearly show that the mass spectral signature of the PSL core for particles with larger SC nodules diminishes to below detection we must conclude that during the ablation process entire particles are consistently evaporated.

Finally, an examination of the $\mathrm{Na}^{+}$signal intensity distributions for particles of all SC nodule sizes reveals that each of the individual particle spectra has significant $\mathrm{Na}^{+}$mass spectral peak intensity, despite the fact that the SC nodule in many of these particles must have been in the PSL particle "shadow."

A comparison between the observed mass spectral intensities at the two laser powers also leads us to conclude that the observed differences between the two laser powers most likely reflect the fact that, at higher laser powers, the ions produced from the PSL core fragments have higher kinetic energy and thus higher probability to escape the ablation plume and avoid the collision based processes of charge transfer and ion suppression by $\mathrm{Na}$.

\section{PSL Particles with Rounded Sodium Lauryl Sulfate (SLS) Containing Nodules}

A suspension of PSL particles contains $\mathrm{Na}$ in two forms. The PSL sphere surface is derivatized with sodium sulfonate groups, which is equivalent to a monolayer. In addition, the suspension of PSL spheres contains among other additives SLS, used to keep the particles dispersed (Reents \& Ge 2000). During evaporation of the suspension-solvent the non-volatile additives stick to the PSL particles forming a Na-containing coating. Since the composition and exact properties of this coating are proprietary we do not know the true density of the coating and will therefore rely only on the measured particle $d_{v a}$.

Observed asphericity of aerosolized PSL particles has been previously reported by Huffman et al. (2005). In a recent paper utilizing SPLAT (Zelenyuk et al. 2005) we have demonstrated, using as an example $302 \mathrm{~nm}$ PSL particles, that PSL asphericity can be explained by the presence of nodules composed of the manufacturer introduced additives mentioned above. The micrographs presented in Figure 12 provide an obvious indication of the presence of rounded nodules. In this and other studies we found a clear correlation between the emergence of the shoulder on the larger diameter side in the PSL $d_{v a}$ distributions and the appearance of PSL particles with observable nodules in the SEM images. We use the presence of SLS to generate our third morphologically distinct Na-containing coated PSL particle type, which, similar to SC, forms localized Na-containing nodules. In order to avoid having the data be distorted by PSL agglomerates we conduct the present study on a relatively dilute PSL suspension and terminate the study at $272 \mathrm{~nm}$, which is lower than $276 \mathrm{~nm}$ - the vacuum aerodynamic diameter of the doublets of 240 nm PSL spheres (Zelenyuk et al. 2006).

Figure 12 shows two low laser power mass spectra of $240 \mathrm{~nm}$ PSL particles. In Figure 12a we present an average of individual particle mass spectra of particles with $d_{v a}$ between $247 \mathrm{~nm}$ and $255 \mathrm{~nm}$. These spectra are dominated by nearly pure PSL particles but the presence of sodium is clearly indicated by the $\mathrm{Na}^{+}$-containing peaks. The spectrum in Figure $12 \mathrm{~b}$ is of larger particles $\left(255 \mathrm{~nm}<d_{v a}<270 \mathrm{~nm}\right)$. These larger particles contain 

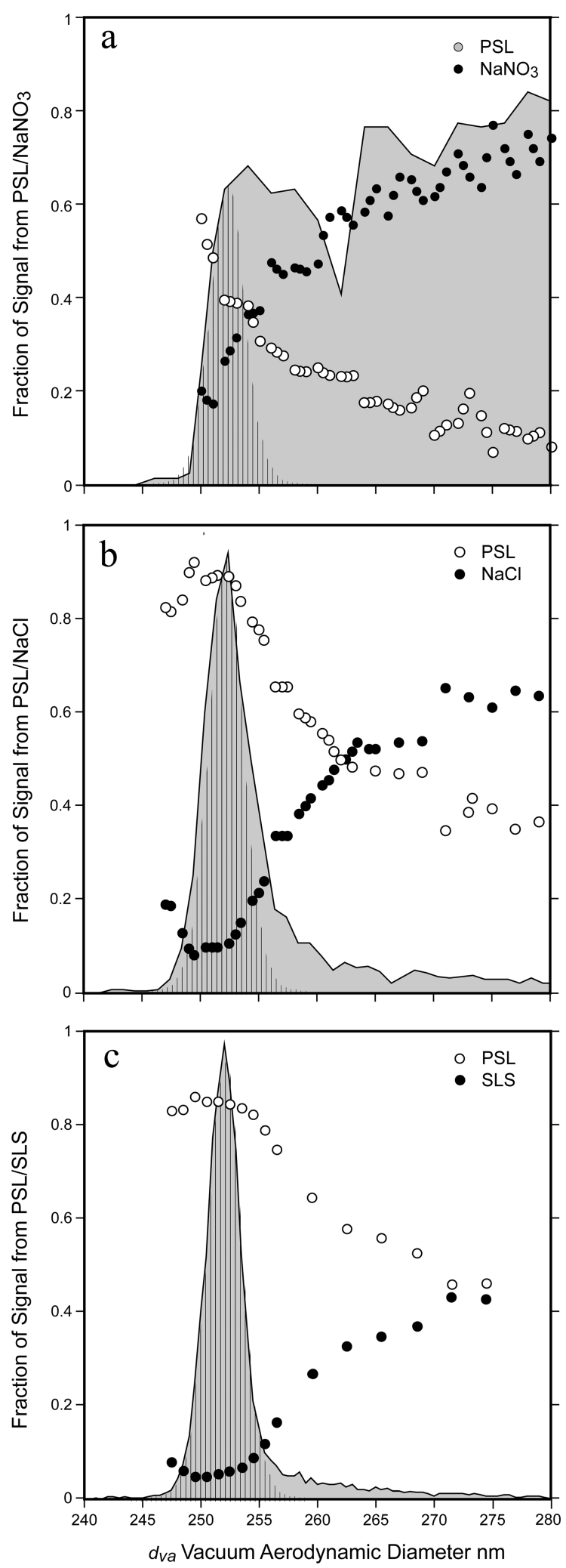

FIG. 13. High resolution (1 to $2 \mathrm{~nm}$ ) comparison between the three PSL coated particle systems presented in this study. The figure shows the fractional mass spectral peak intensities superimposed on the vacuum aerodynamic size distributions of (a) $\mathrm{NaNO}_{3}$, (b) $\mathrm{NaCl}$, and (c) SLS coated PSL particles. See text for detailed description. larger SLS nodules and yield mass spectra dominated by $\mathrm{Na}^{+}-$ containing peaks. Mass spectra obtained at high laser power exhibit higher degree of fragmentation and relatively more intensity in the PSL mass spectral peaks.

Figure $13 \mathrm{c}$ below is a plot of the integrated mass spectral signals assigned to the PSL core and to the SLS-containing nodules as a function of $d_{v a}$. These data are superimposed on the measured $d_{v a}$ distribution for this study. At smaller $d_{v a}$ most PSL particles are virtually devoid of SLS and their mass spectral signature is dominated by PSL. Particles with $d_{v a}$ larger than $255 \mathrm{~nm}$ have significant SLS-containing nodules. Note that the increase in the $\mathrm{Na}^{+}$based mass spectral signal intensity is accompanied by a decrease in the PSL signal. This trade-off in intensities is consistent with a charge exchange, in which $\mathrm{Na}^{+}$ions are formed at the expense of carbonaceous ions. As in the other systems, the relative intensities of PSL core and SLS-containing coating are a function of laser power. At lower laser power (not shown) the SLS mass spectral signal intensity is relatively higher for all particle sizes.

\section{SUMMARY AND CONCLUSION}

We summarize this paper with a high resolution comparative view of the mass spectral behavior of PSL particles coated with Na-containing compounds as the first few nanometers of coating are added. Figure 13 presents on an expanded scale of the measured $d_{v a}$ distributions for the three datasets. The shaded areas indicate the observed $d_{v a}$ distributions and the striped ones mark the $d_{v a}$ distribution of pure $240 \mathrm{~nm}$ PSL particles. Superimposed on top of the observed $d_{v a}$ distributions are the measured mass spectral intensities expressed as the fractions of integrated peak intensities that are assigned to either the PSL spheres or to the Na-containing coating.

The observed $d_{v a}$ distributions of the SN and SC coated PSL particles are significantly different as a result of particle asphericity. In contrast the two $d_{v a}$ distributions of particles with asymmetric nodules are found to be qualitatively similar.

Comparing the behavior of the mass spectral peak intensities of three particle types we find first that even when PSL particles are coated by sufficient amount of SC or SN to account for more than $50 \%$ of the particle more than half of the coated PSL particles can be detected by ablation based single particle mass spectrometry. But, the PSL signal from completely encapsulated particles decays faster than from particles with localized nodules.

In general the data indicate that the presence of the alkali metal suppresses the PSL core ions and the degree to which this takes place depends on the relative amount of Na-containing compound and on its spatial distribution on the PSL sphere surface, i.e., the morphology of the coated particle. Particles of similar morphologies exhibit remarkably similar behavior, which is distinct from that observed for the encapsulated PSL spheres.

The data presented for these three systems clearly illustrates some of the difficulties in turning ablation based single 
particle mass spectrometry into a quantitative tool. We find that for thinner coatings, in which the PSL core is detectable, the relative mass spectral intensities of the peaks assigned to the $\mathrm{Na}$-containing coatings and the PSL core are not linearly related to the amount of these substances in the particles. They are also strong functions of laser power and the details of particle morphology. We conclude that the decrease in PSL mass spectral signal is a result of ion suppression.

The data presented in this paper also support the conclusion that at the laser powers and wavelengths deployed in this study the ablation laser evaporates the entire particle for all particle sizes used here.

\section{REFERENCES}

Buzorius, G., Zelenyuk, A., Brechtel, F., and Imre, D. (2002). Simultaneous Determination of Individual Ambient Particle Size, Hygroscopicity and Composition, Geophys. Res. Lett. 29(20):1974, doi:10.1029/2001GL014221.

Carson, P. G., Johnston, M. V., and Wexler, A. S. (1997). Real-Time Monitoring of the Surface and Total Composition of Aerosol Particles, Aerosol Sci. Technol. 26:291-300.

Chung, S. H., and Seinfeld, J. H. (2002). Global Distribution and Climate Forcing of Carbonaceous Aerosols, J. Geophys. Res. 107(D19):4407, doi:10.1029/2001JD001397.

Fuller, K. A., Malm, W. C., and Kreidenweis, S. M. (1999). Effects of Mixing on Extinction by Carbonaceous Particles, J. Geophys. Res. 104:15941-15954.

Ge, Z. Z., Wexler, A. S., and Johnston, M. V. (1996). Multicomponent Aerosol Crystallization, J. Colloid Interface Sci. 183:68-77.

Ge, Z. Z., Wexler, A. S., and Johnston, M. V. (1998). Laser Desorption/Ionization of Single Ultrafine Multicomponent Aerosols, Environ. Sci. Technol. 32:3218-3223.

Gross, D. S., Galli, M. E., Silva, P. J., and Prather, K. A. (2000). Relative Sensitivity Factors for Alkali Metal and Ammonium Cations in Single Particle Aerosol Time-of-Flight Mass Spectra, Anal. Chem. 72(2):416422.

Guazzotti, S. A., Whiteaker, J. R., Suess, D., Coffee, K. R., and Prather, K. A. (2001). Real-Time Measurements of the Chemical Composition of SizeResolved Particles during a Santa Ana Wind Episode, California USA, Atmos. Environ. 35:3229-3240.

Hoffman R. C., Laskin A., and Finlayson-Pitts, B. J. (2004). Sodium Nitrate Particles: Physical and Chemical Properties during Hydration and Dehydration, and Implications for Aged Sea Salt Aerosols, J. Aerosol Sci. 35(7):869887.

Huffman, J. A., Jayne, J. T., Drewnick, F., Aiken, A. C., Onasch, T., Worsnop, D. R., and Jimenez, J. L. (2005). Design, Modeling, Optimization, and Experimental Tests of a Particle Beam Width Probe for the Aerodyne Aerosol Mass Spectrometer, Aerosol Sci. Technol. 39:1143-1163.

Jacobson, M. Z. (2000). A Physically-Based Treatment of Elemental Carbon Optics: Implications for Global Direct Forcing of Aerosols, Geophys. Res. Lett. 27:217-220.

Jacobson, M. Z. (2001). Strong Radiative Heating due to the Mixing State of Black Carbon in Atmospheric Aerosols, Nature 409: 695-697.

Katrib, Y., Martin, S. T., Hung, H. M., Rudich, Y., Zhang, H. Z., Slowik, J. G., Davidovits, P., Jayne, J. T., and Worsnop, D. R. (2004). Products and Mechanisms of Ozone Reactions with Oleic Acid for Aerosol Particles Having Core-Shell Morphologies, J. Phys. Chem. A 108(32):6686-6695.
Katrib, Y., Martin, S. T., Rudich, Y., Davidovits, P., Jayne, J. T., and Worsnop, D. R. (2005). Density Changes of Aerosol Particles as a Result of Chemical Reaction, Atmos. Chem. Phys. 5:275-291.

Knochenmuss, R., and Zenobi, R. (2003). MALDI Ionization: The Role of InPlume Processes, Chem. Rev. 103:441-452.

Lake, D. A., Tolocka, M. P., Johnston, M. V., and Wexler, A. S. (2004). The Character of Single Particle Sulfate in Baltimore, Atmos. Environ. 38:53115320.

Laskin, A., Cowin, J. P., and Jedema, M. J. (2005). "Off-line Analysis of Individual Environmental Particles using Modern Methods of Electron Microscopy and X-ray Microanalysis," J. Electron Spectr. Rel. Phen. (in press).

Lee, S. H., Murphy, D. M., Thomson, D. S., and Middlebrook, A. M. (2002). Chemical Components of Single Particles Measured with Particle Analysis by Laser Mass Spectrometry (PALMS) during the Atlanta Supersite Project: Focus on Organic/Sulfate, Lead, Soot, and Mineral Particles, J. Geophys. Res. 107(D1), 4003, doi:10.1029/2000JD000011.

Lesins, G., Chylek, P., and Lohmann, U. (2002). A Study of Internal and External Mixing Scenarios and its Effect on Aerosol Optic Properties and Direct Radiative Forcing, J. Geophys. Res. 107(D10), 4094, doi:10.1029/2001JD000973.

Reents, W. D., and Ge, Z. Z. (2000). Simultaneous Elemental Composition and Size Distributions of Submicron Particles in Real Time Using Laser Atomization/Ionization Mass Spectrometry, Aerosol Sci. Technol. 33:122-134.

Reilly, P. T. A., Lazar, A. C., Gieray, R. A., Whitten, W. B., and Ramsey, J. M. (2000). The Elucidation of Charge-Transfer-Induced Matrix Effects in Environmental Aerosols Via Real-Time Aerosol Mass Spectral Analysis of Individual Airborne Particles, Aerosol Sci. Technol. 33:135-152.

Schoolcraft, T. A., Constable, G. S., Shigilei, L. V., and Garrison, B. J. (2000). Molecular Dynamics Simulation of the Laser Disintegration of Aerosol Particles, Anal. Chem. 72:5143-5150.

Tang I. N., and Munkelwitz, H. R. (1994). Water Activities, Densities, and Refractive- Indexes of Aqueous Sulfates and Sodium-Nitrate Droplets of Atmospheric Importance, J. Geophys. Res. Atmos. 99(D9):18801-18808.

Vera, C. C., Trimborn, A., Hinz, K.-P., and Spengler, B. (2005). Initial Velocity Distributions of Ions Generated by In-Flight Laser Desorption/Ionization of Individual Polystyrene Latex Microparticles as Studied by the Delayed Ion Extraction Method, Rapid Comm. Mass Spectrom. 19:133-146.

Weiss, M., Verheijen, P. J. T., Marijnissen, J. C. M., and Scarlett, B. (1997). On the Performance of an on Line Time-of-flight Mass Spectrometer for Aerosols, J. Aerosol Sci. 28:159-171.

Wittig, A. E., Anderson, N., Khlystov, A. Y., Pandis, S. N., Davidson, C., and Robinson, A. L. (2004). Pittsburgh Air Quality Study Overview, Atmos. Environ. 38:3107-3125.

Woods, E. III, Smith, G. D., Miller, R. E., and Baer, T. (2002). Depth Profiling of Heterogeneously Mixed Aerosol Particles Using Single-Particle Mass Spectrometry, Anal. Chem. 74:1642-1649.

Zelenyuk, A., and Imre, D. (2005). Single Particle Laser Ablation Time-offlight Mass Spectrometer: An introduction to SPLAT, Aerosol Sci. Technol. 39(6):554-568.

Zelenyuk, A., Cai, Y., and Imre, D. (2006). From Agglomerates of Spheres to Irregularly Shaped Particles: Determination of Dynamic Shape Factors from Measurements of Mobility and Vacuum Aerodynamic Diameters. Aerosol Sci. Technol. 40:197-217.

Zelenyuk, A., Cai, Y., Chieffo, L., and Imre, D. (2005). High Precision Density Measurements of Single Particles: The Density of Metastable Phases, Aerosol Sci. Technol. 39(10):972-986.

Zhang, Q., Canagaratna, M. R., Jayne J. T., and Worsnop, D. R., and Jimenez, J. L. (2005). Time- and Size-Resolved Chemical Composition of Submicron Particles in Pittsburgh: Implications for Aerosol Sources and Processes, J. Geophys. Res. 110, D07S09, doi:10.1029/2004JD004649. 